\title{
Continuous Saline Bladder Irrigation in Reducing Recurrence and Progression When Compared to Immediate Mitomycin- C Instillation Post- Resection of Bladder Tumor: A Short Communication
}

\author{
Morad Bani-Hani ${ }^{\text {* }}$, Ammar Abdel Majid ${ }^{2}$, Mohammad Talal Al-Zubi ${ }^{3}$, Saddam \\ Al Demour ${ }^{4}$, Mazhar Salim Al Zoubi ${ }^{5}$, Mohannad Abuhamad ${ }^{6}$, Abdallah \\ Santarisi $^{6}$, Batool Taysir Eldos ${ }^{7}$, Jad Alsmadi ${ }^{1}$, Basel Elayan ${ }^{8}$
}

\begin{abstract}
Introduction: Intravesical chemotherapy instillation by mitomycin - C (MMC) immediately after transurethral resection of bladder tumor (TURBT), although effective in reducing the incidence of non- muscle invasive bladder cancer (NMIBC) recurrence, can result in non desirable effects like bladder irritation and hematuria . Continuous bladder irrigation with saline post resection has been studied as an alternative. In our study we compare the rates of NMIBC recurrence and progression in patients who were treated with either MMC or CSBI immediately after tumor resection. Methods: We retrospectively reviewed the medical records of patients with NMIBC at our institution in Jordan university hospital in the period between 2015-2019. Postoperative instillation of MMC or CSBI for four hours was recorded. Follow up of the patients for recurrence or progression in the first 2 years after diagnosis was recorded and compared for both groups. Results: One hundred nineteen patients met inclusion criteria. Fifty four patients received MMC and 65 patients received CSBI immediately post TURBT. Kaplan-Meieranalysis for recurrence- free survival and progression- free survival didn't show a significant difference between both groups with P-value 0.88 and 0.14 respectively. Conclusion: Postoperative CSBI for four hours following tumor resection is equivalent to immediate postoperative MMC instillation for NMIBC in terms of recurrence or progression rates with fewer side effects. However further research is needed in this field .
\end{abstract}

Keywords: TCC- NMIBC- Irrigation- TURT

Asian Pac J Cancer Prev, 23 (1), 171-175

\section{Introduction}

Bladder cancer showed different stages during its development and non-muscle invasive bladder cancer (NMIBC) represented about $70 \%$ of all bladder cancers (Clark et al., 2013). NMIBC is also known as superficial bladder cancer due to its histological features by not invading the bladder muscular tissues. At the pathological level, the NMIBC shows better prognosis compared to advanced stages of bladder cancers.

Therefore, earlier diagnosis and treatment showed significant improvement in the prognosis of the NMIBC. For instance, transurethral resection of bladder tumor (TURBT) is considered as a gold standard treatment for the NMIBC, however, a risk of recurrence and progression has been reported after resection (Witjes, 2009). Therefore, frequent, and intensive follow-up is highly recommended for early pick up and treatment of NMIBC recurrence after resection (Chamie et al., 2011; James and Gore, 2013). It is believed that the post-TURBT cancerous cells can start the re-implantation process to nearby urinary bladder tissues which explain the recurrence of NMIBC (Babjuk et al., 2017). Accordingly, the standard adjuvant therapy following TURBT for NMIBC is intravesical chemotherapy like mitomycin-C (MMC). Different reports have shown a reduction in the NMIBC recurrence after immediate MMC intravesical instillation. (Sylvester et al., 2016; Sylvester et al., 2004). Reduction in tumor

${ }^{1}$ Department of Special Surgery, Division of Urology, School of Medicine, University of Hashemite University, Zarka, Jordan. ${ }^{2}$ Al sleibi, Clinical Sciences, Al-Balqa Applied University, Jordan. ${ }^{3}$ Department of Surgery, Division of Urology, School of Medicine, Yarmouk University, Irbid, Jordan. ${ }^{4}$ Department of Special Surgery, Division of Urology, School of Medicine, University of Jordan, Amman. ${ }^{5}$ Department of Basic Medical Sciences, Faculty of Medicine, Yarmouk University, Irbid, Jordan. ${ }^{6} J o r d a n$ University Hospital, Amman, Jordan. ${ }^{7}$ Department of Clinical Sciences, School of Medicine, Yarmouk university, Irbid, Jordan. ${ }^{8}$ Faculty of Medicine, Yarmouk University, Irbid, Jordan.*For Correspondence: Morad_bh@hu.edu.jo 
recurrence has been attributed to the prevention of cancerous cells from re-implantation by MMC (Doherty et al., 1999). Nonetheless, several urologists have certain restrictions to apply the intravesical instillation post TURBT due to the need for distinct postsurgical care, in addition, some lower urinary tract complications have been raised like irritability, hematuria, dysuria and frequency caused by chemotherapy treatment (Cookson et al., 2012; Koya et al., 2006). Therefore, an alternative treatment option or strategy is required for certain patients to overcome the side effects and complications that have been associated with the available chemotherapies like MMC (Jones et al., 2012).

As an alternative option, quite a few researchers have suggested the use of sterile water/saline irrigation to reduce NMIBC recurrence by lyse-preventive mechanism against the free-floating cancerous cells from re-implantation (Onishi et al., 2011; Taoka et al., 2015). Previously, in a retrospective study, the researchers demonstrated a preventive effect of Continuous saline bladder irrigation (CSBI) on the recurrence of NMIBC post-TURBT (Onishi et al., 2011). Moreover fewer studies have investigated the effect of CSBI on post-TURBT NMIBC recurrence rate, showing that $\mathrm{CSBI}$ seems to be better in prevention of recurrence with lesser local toxicities (Do et al., 2018; Zhou et al., 2019).

Consequently, the current study aimed to evaluate the effect of the CSBI of the isotonic normal-saline post-TURBT on the recurrence or progression in NMIBC patients compared to the single instillation of MMC chemotherapy regimen.

\section{Materials and Methods}

\section{Study design}

As a retrospective study, the experimental procedure and data collection were conducted from January 2015 to April 2019 at our institution in Jordan university hospital after obtaining the ethical approval which was provided by the Faculty of Medicine and The Deanship of Scientific Research and Graduate Study at Jordan university.

\section{Population}

The study population included patients diagnosed with NMIBC who underwent TURBT in the period between January 2015 to April 2019.

\section{Inclusion and exclusion criteria}

Inclusion criteria were to include all patients diagnosed with NMIBC post TURBT for transitional cell carcinoma. On the other hand, all patients that showed squamous cell, adenocarcinoma, small cell, sarcomatoid, and micropapillary disease were excluded from the study. In addition, all patients diagnosed with muscle-invasive bladder urothelial carcinoma (T2), tumor resected incompletely, known metastatic disease, concomitant upper urinary tract urothelial carcinoma, and bladder perforation post-TURBT were also excluded from the study.

\section{Design}

The study population information was retrospectively retrieved and reviewed from the medical record. After reviewing the pathological and clinical data, only individuals who received either CSBI or MMC immediately post TURBT were selected to be included in the study. On the other hand, all individuals with bladder perforation or who did not receive CSBI or MMC immediately post resection were excluded from the study.

$\mathrm{MMC}$ instillation was given at $40 \mathrm{mg} / 50 \mathrm{~mL}$ of saline and kept inside the urinary bladder for $60-90 \mathrm{~min}$ by Foley catheter clamping, followed by the drain, while the catheter was kept if considered necessary. In the other arm, the CSBI was performed if either chemotherapy was not available post resection or contraindicated to be given like gross hematuria post TURBT, in these patients CSBI was cosidered by placement of a three-way Foley catheter and left running using isotonic solution $(0.9 \% \mathrm{NaCl})$ for approximately four hours post-TURBT. If there is no need, all patients were discharged home on the same day after catheter removal and successful trial of the void without a catheter.

Patients were Follow up by cystoscopy for recurrence and progression for at least 3 months post first resection (range 3-24 months) and recorded. Tumor recurrence was considered when the pathologist reported and confirmed the presence of bladder urothelial carcinoma with the same grade/stage on resected biopsies. In addition, the recurrence was reported if the cystoscopy showed small visible lesions which were treated by coagulation without biopsy. Moreover, the progression was reported by pathologists if there was an increase in grade or stage of the disease. All patients received either intravesical chemotherapy or immunotherapy (BCG) later on as adjuvant treatment.

\section{Statistical Analysis}

The statistical analysis was performed using SPSS 20.0 software to calculate the differences between patients' arms by t-test and chi-square tests. Kaplan-Meier curve was generated to estimate the recurrence-free and progression-free survival and compared by log-rank test. The significant value was considered when the $\mathrm{p}$-value less than 0.05 .

\section{Results}

The study population included 119 individuals who underwent TURBT for NMIBC during the period between January 2015 to April 2019 at our institution and met all inclusion criteria were divided into two groups according to immediate post-resection therapy, the first group of 54 patients $(45 \%)$ received intravesical chemotherapy immediately post TURBT while the second group 65 patients $(55 \%)$ received CSBI for at least four hours post TURBT.

The mean age was 60.8 and 61.3 years for both groups respectively with a $\mathrm{P}$-value was 0.835 . In general, about $90 \%$ of patients were male, result close to Al Khader et al result (Al Khader et al., 2019), while if we take it by group, $96.3 \%$ of the patients who underwent MMC instillation 
Table 1. Patient and Primary Tumor Characteristics Stratified by Post-Resection Treatment

\begin{tabular}{|c|c|c|c|c|}
\hline & & \multicolumn{2}{|c|}{ Groups } & \multirow[t]{2}{*}{$P$ value } \\
\hline & & Irrigation & Chemotherapy & \\
\hline Mean age & & 61.292 & 60.87 & 0.835 \\
\hline \multirow[t]{2}{*}{ Gender } & Male & $58(89.2 \%)$ & $52(96.3 \%)$ & 0.147 \\
\hline & Female & $7(10.8 \%)$ & $2(3.7 \%)$ & \\
\hline \multirow[t]{2}{*}{ Smoking } & No & $15(23.1 \%)$ & $5(9.3 \%)$ & 0.045 \\
\hline & Yes & $50(76.9 \%)$ & $49(90.7 \%)$ & \\
\hline \multirow[t]{3}{*}{ Initial tumor T } & $\mathrm{Ta}=1$ & $37(56.9 \%)$ & $39(72.2 \%)$ & 0.093 \\
\hline & $\mathrm{T} 1=2$ & $28(43.1 \%)$ & $14(25.9 \%)$ & \\
\hline & $\mathrm{CIS}=3$ & $0(0.0 \%)$ & $1(1.9 \%)$ & \\
\hline \multirow[t]{2}{*}{ Initial tumor grade } & Low Grade & $38(58.5 \%)$ & $39(72.2 \%)$ & 0.118 \\
\hline & High Grade & $27(41.5 \%)$ & $15(27.8 \%)$ & \\
\hline
\end{tabular}

Table 2. Means for Recurrence-Free Time for Different Groups

\begin{tabular}{lcccc}
\hline Groups & & \multicolumn{2}{c}{ Mean } \\
& Estimate & Std. Error & Lower Bound & Upper Bound \\
\hline irrigation & & 1.235 & 11.665 & 16.504 \\
chemotherapy & 14.084 & 1.423 & 10.231 & 15.809 \\
Overall & 13.02 & 0.929 & 11.771 & 15.411 \\
\hline
\end{tabular}

immediately post-resection were male compared to $89.2 \%$ for the patients received CSBI post resection with $\mathrm{P}$-value $=0.147$. when assessing smoking as a risk factor for bladder cancer we can see that most of the patients in both groups are smokers ( $90.7 \%$ and $76.9 \%$ respectively).

Regarding tumor characteristics, for stage, most of the tumor in both groups are limited to the mucosa (Ta) at the initial pathology at the time of diagnosis $(72.2 \%$ for the first group and $56.9 \%$ for the second group with a P-value of 0.93 ), regarding the grade, about $72.2 \%$ of those who received MMC post TURBT and $58.5 \%$ of patients received saline irrigation post-resection have a low grade as the primary grade at the time of diagnosis with $\mathrm{P}$-value $=0.118$. Table 1 summarizes the patient characteristics (age, sex and smoking status) in addition to primary tumor characteristics including stage and grade for both groups.

The follow-up period extended to a period of 24 months for most individuals, recurrences were observed in $63 \%(75 / 119)$ of all cases. The recurrence free rate at 3, 12 and 24 months for the irrigation group were $60 \%, 47 \%, 36 \%$ respectively, while In chemotherapy group, recurrence-free rate were $50 \%, 46 \%, 35 \%$ respectively. As shown in Table 2, Kaplan-Meire analysis of recurrence-free survival did not show any significant difference between the groups (log rank test: $\mathrm{P}=0.80$ ).

On the other hand, Progression were observed in $8 \%$ (10/119) of all cases. The progression free rate at 3, 12 and 24 months for the irrigation group were 93\%, 92\%, $87 \%$ respectively. In chemotherapy group, progression -free rate at 3, 12 and 24 months were 98\%, 96\%, 100\% respectively.

Interestingly, the Kaplan-Meier analysis of progression-free survival in Table 3 showed no significant difference between the saline irrigated group and the chemotherapy-treated group as shown in Table 2 (log-rank test: $\mathrm{P}=0.1$ ).

After performing a survival analysis and because the number of subjects are small and number of events (death) are small as well, the survival may not be the best outcome for this analysis, we have decided to turn this work to a short communication.

\section{Discussion}

The NMIBC recurrence rate is one of the most common features of this bladder cancer subtype after TURBT. Therefore, preventive treatments and measures are required to reduce this recurrence and progression post TURBT. For instance, Gudjónsson et al demonstrated

Table 3. Means for Progression-Free Time for Different Groups

\begin{tabular}{lcccc}
\hline Groups & Estimate & Std. Error & \multicolumn{2}{c}{ 95\% Confidence Interval } \\
& & & Lower Bound & Upper Bound \\
\hline irrigation & 22.517 & 0.693 & 21.159 & 23.875 \\
chemotherapy & 23.38 & 0.444 & 22.509 & 24.251 \\
Overall & 22.909 & 0.431 & 22.063 & 23.754 \\
\hline
\end{tabular}


a significant inhibitory effect of MMC on the cancer recurrence after TURBT in NMIBC patients (Gudjónsson et al., 2009). Additionally, a meta-analysis study showed that immediate instillation of intravesical chemotherapy reduced tumor recurrence compared to untreated NMIBC patients (Sylvester et al., 2004). Intravesical chemotherapy and immunotherapy, have displayed an effective reduction in the recurrence and progression of NMIBC post TURBT respectively. Another study showed a reduction in disease recurrence rate from about 59\% to $45 \%$ in 5 years after subjecting patients to instillation of intravesical chemotherapy (Sylvester et al., 2016). Hence, the American Urological Association (AUA) as well as European Association of Urology (EAU) guidelines currently recommended performing instillation of intravesical chemotherapy in NMIBC patients post-TURBT (Babjuk et al., 2017; Chang et al., 2017).

However, the risk of side effects such as gross hematuria, bladder irritation, and dysuria cannot be avoided (Sylvester et al., 2004; Sylvester et al., 2002). Therefore, other post-TURBT treatments and measures are needed to avoid the adverse effect of the available chemotherapy treatments. Moskovitz et al., (1987) proposed that distilled-water intravesical irrigation post-TURBT would lead to a reduction in NMIBC recurrences (Moskovitz and Levin, 1987). Then, some studies demonstrated that NMIBC recurrence was significantly similar when the post-TURBT patients are subjected to CSBI or treated with MMC (Bijalwan et al., 2017; Onishi et al., 2011; Onishi et al., 2017). These findings were supported by another study when the researchers showed that sterile water intravesical irrigation has the same effect as an immediate single dose of intravesical MMC with fewer complications (Bijalwan et al., 2017).

Using fluid irrigation as a concept for the extermination of remaining tumor cells is not a new concept after surgical procedures. Surgeons have traditionally used fluid to irrigate surgical sites as a standard protocol for many reasons such as simple washing to remove any unwanted residuals, bacterial dilution which will reduce a load of bacterial load at the site of surgery and lysis of cells including possible floating cancer cells (Whitesideet al., 2005). Several studies have investigated the ideal type of fluid to use for irrigation. For instance, Ito et al. , (2011) proposed the advantage use of water irrigation over saline irrigation after colorectal cancer surgical procedure in mice. The superiority of using distilled water for irrigation post-operative can be attributed to the osmotic cytotoxic of distilled water compared to the isotonic solutions like normal saline.

In our study, we used isotonic solution $0.9 \%$ normal saline to avoid post-operative complications from the hypotonic fluid. The results of the current study are consistent with the previous studies that showed the possible use of normal saline for the irrigation of postTURBT in NMIBC patients with comparable recurrence and progression rate to $\mathrm{MCC}$ treated patients. However, our study has some potential limitations like the relatively small sample size, therefore, more studies are recommended to include a larger number of patients which would strengthen the findings. Secondly, longer follow-up of patients is needed to get more accurate results.

In conclusion, the present study showed that postoperative CSBI for at least four hours post-TURBT is equivalent to immediate postoperative MMC treatment for NMIBC in terms of rates of recurrence or progression with fewer side effects. However further research is needed in this field to determine the duration of irrigation needed and whether other postoperative instillations reduce the recurrence and progression rates with minimal side effects compared to MMC.3.

\section{Author Contribution Statement}

All authors have participated, read, and approved this work.

Morad Bani-Hani: Project Development, Manuscript writing and editing, Data Analysis. Mohammad Talal Al-Zubi: Project Development, Manuscript writing and editing, Data Analysis. Saddam Al Demour: Project Development, Manuscript writing and editing. Maher Salim Al Zoubi: Manuscript writing and editing. Mohannad Abuhamad: Data Collection and analysis. Abdallah Santarisi: Data Collection and analysis Batool. Taysir Elldos: Manuscript writing and editing, Data Analysis. Jad Alsmadi: Manuscript writing and editing, Data Analysis. Ammar Abdel Majid Al Sleibi: Manuscript writing and editing, Data Analysis. Basel Elayan: Manuscript writing and editing, Data Analysis.

\section{Acknowledgements}

None.

\section{Ethical approval}

This work was approved by the Ethical Committee at Jordan University Hospital, Amman, Jordan. IRB number: 1012020116050 .

\section{Availability of data}

Available upon reasonable request.

\section{Conflicts of interest}

None.

\section{References}

Al Khader AM, Abu Shahin NI, Obeidat FN, Al-Chalabi MA (2019). Urinary bladder cancer in Jordanian adults: A histopathological and epidemiological study from a tertiary care center in Amman. J Pak Med Assoc, 69, 415-7.

Babjuk M, Böhle A, Burger M, et al (2017). EAU Guidelines on Non-Muscle-invasive Urothelial Carcinoma of the Bladder: Update 2016. Eur Urol, 71, 447-61.

Bijalwan P, Pooleri GK, Thomas A (2017). Comparison of sterile water irrigation versus intravesical mitomycin $\mathrm{C}$ in preventing recurrence of nonmuscle invasive bladder cancer after transurethral resection. Indian J Urol, 33, 144-8.

Chamie K, Saigal CS, Lai J, et al (2011). Compliance with guidelines for patients with bladder cancer: variation in the delivery of care. Cancer, 117, 5392-401. 
Chang SS, Bochner BH, Chou R, et al (2017). Treatment of NonMetastatic Muscle-Invasive Bladder Cancer: AUA/ASCO/ ASTRO/SUO Guideline. J Urol, 198, 552-9.

Clark PE, Agarwal N, Biagioli MC, et al (2013). Bladder cancer. J Natl Compr Canc Netw, 11, 446-75.

Cookson MS, Chang SS, Oefelein MG, et al (2012). National practice patterns for immediate postoperative instillation of chemotherapy in nonmuscle invasive bladder cancer. J Urol, 187, 1571-6.

Do J, Lee SW, Jeh SU, et al (2018). Overnight continuous saline irrigation after transurethral resection for non-muscleinvasive bladder cancer is helpful in prevention of early recurrence. Can Urol Assoc J, 12, E480-e3.

Doherty AP, Trendell-Smith N, Stirling R, et al (1999). Perivesical fat necrosis after adjuvant intravesical chemotherapy. BJU Int, 83, 420-3.

Gudjónsson S, Adell L, Merdasa F, et al (2009). Should all patients with non-muscle-invasive bladder cancer receive early intravesical chemotherapy after transurethral resection? The results of a prospective randomised multicentre study. Eur Urol, 55, 773-80.

Ito F, Camoriano M, Seshadri M, et al (2011). Water: a simple solution for tumor spillage. Ann Surg Oncol, 18, 2357-63.

James AC, Gore JL (2013). The costs of non-muscle invasive bladder cancer. Urol Clin North Am, 40, 261-9.

Jones G, Cleves A, Wilt TJ, et al (2012). Intravesical gemcitabine for non-muscle invasive bladder cancer. Cochrane Database Syst Rev, 1, Cd009294.

Koya MP, Simon MA, Soloway MS (2006). Complications of intravesical therapy for urothelial cancer of the bladder. J Urol, 175, 2004-10.

Moskovitz B, Levin DR (1987). Intravesical irrigation with distilled water during and immediately after transurethral resection and later for superficial bladder cancer. Eur Urol, 13, 7-9.

Onishi T, Sasaki T, Hoshina A, et al (2011). Continuous saline bladder irrigation after transurethral resection is a prophylactic treatment choice for non-muscle invasive bladder tumor. Anticancer Res, 31, 1471-4.

Onishi T, Sugino Y, Shibahara T, et al (2017). Randomized controlled study of the efficacy and safety of continuous saline bladder irrigation after transurethral resection for the treatment of non-muscle-invasive bladder cancer. BJU Int, 119, 276-82.

Sylvester RJ, Oosterlinck W, Holmang S, et al (2016). Systematic review and individual patient data metaanalysis of randomized trials comparing a single immediate instillation of chemotherapy after transurethral resection with transurethral resection alone in patients with stage pTa-pT1 urothelial carcinoma of the bladder: Which Patients Benefit from the Instillation?. Eur Urol, 69, 231-44.

Sylvester RJ, Oosterlinck W, van der Meijden AP (2004). A single immediate postoperative instillation of chemotherapy decreases the risk of recurrence in patients with stage $\mathrm{Ta}$ T1 bladder cancer: a meta-analysis of published results of randomized clinical trials. $J$ Urol, 171, 2186-90, quiz 435.

Sylvester RJ, van der MA, Lamm DL (2002). Intravesical bacillus Calmette-Guerin reduces the risk of progression in patients with superficial bladder cancer: a meta-analysis of the published results of randomized clinical trials. $J$ Urol, 168, 1964-70.

Taoka R, Williams SB, Ho PL, et al (2015). In-vitro cytocidal effect of water on bladder cancer cells: The potential role for intraperitoneal lavage during radical cystectomy. Can Urol Assoc J, 9, E109-13.

Whiteside OJ, Tytherleigh MG, Thrush S, et al (2005). Intraoperative peritoneal lavage--who does it and why? Ann $R$
Coll Surg Engl, 87, 255-8.

Witjes JA (2009). Topic issue on new treatments in bladder cancer. World J Urol, 27, 285-7.

Zhou Z, Zhao S, Lu Y, et al (2019). Meta-analysis of efficacy and safety of continuous saline bladder irrigation compared with intravesical chemotherapy after transurethral resection of bladder tumors. World J Urol, 37, 1075-84.

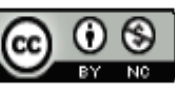

This work is licensed under a Creative Commons AttributionNon Commercial 4.0 International License. 